Aceptado: Junio 2018

\title{
La satisfacción de los usuarios de actividades dirigidas de los Centros deportivos municipales de Barcelona
}

\section{Customer satisfaction in directed activities in municipal sports centers}

\author{
Pilar Aparicio Chueca*, Amal Elasri Ejjaberi y Xavier M. Triadó Ivern
}

Departamento de Empresa. Facultad de Economía y Empresa. Universidad de Barcelona

\begin{abstract}
Resumen: El objetivo de este trabajo es el de identificar y analizar los factores que subyacen en el nivel de satisfacción de los usuarios de centros deportivos municipales que realizan actividades dirigidas y compararlo con aquellos usuarios que no hacen uso de este servicio. Los datos fueron recogidos de una encuesta entre los clientes de centros deportivos municipales, de los que 536 sujetos manifestaron realizar actividades dirigidas en el centro. Las mujeres y los usuarios con un nivel de estudios secundarios son los que más utilizan estos servicios. Un análisis factorial aplicado revela tres dimensiones que contribuyen a la satisfacción, en ambos casos, pero la composición de cada uno de ellos es diferente. Finalmente, del análisis de regresión múltiple se desprende que un gerente debe prestar atención, en primer lugar, a la calidad de las instalaciones y la comunicación ya que fueron el mejor predictor de la satisfacción.

Palabras clave: Satisfacción, actividades dirigidas, centros deportivos
\end{abstract}

Abstract: The aim of this work is to identify and analyze the factors that underlie the level of satisfaction of users of municipal sports centers that conduct directed activities and compare it with those users who do not use this service. Data were collected in a survey among users of municipal sports centers, of which 536 subjects stated that they carried out directed activities at the center. Women and users with a high school level are the ones who use these services the most. An applied factor analysis reveals three dimensions that contribute to satisfaction, in both cases, but the composition of each of them is different. Finally, the multiple regression analysis shows that a manager must pay attention, first of all, to the quality of the facilities and communication since they were the best predictor of satisfaction.

Key words: Satisfaction, directed activities, sports centers.

\section{Introducción}

La gestión de la satisfacción que percibe el cliente es una forma indirecta de gestionar la calidad en el sector servicios, también en las empresas del sector deportivo. El concepto de satisfacción deviene importante en todas las áreas de estudio, ya que si los clientes sienten satisfacción por una marca o producto, realizarán con mayor probabilidad compras repetitivas y futuras recomendaciones del mismo (Cronin, Brady y Hult, 2000). La satisfacción ha sido, por tanto, considerada como un componente crucial para el éxito de los centros deportivos, en el que Bodet (2006) consideraba el término como un concepto clave, tanto para gestores como para investigadores en el área de servicios.

Las actividades dirigidas en los centros deportivos son cada vez más amplias y más demandadas por los clientes (Celestino y Biencinto, 2012), convirtiéndose en un punto clave para la estrategia de fidelización de estos centros (Baena, García, Bernal, Lara y Gálvez, 2016). Investigaciones previas confirman la importancia de contar con una elección adecuada de técnicos deportivos que permitan al cliente recibir una atención lo más adecuada y personalizada posible (Baena et al., 2016; Nuviala, Tamayo, Iranzo y Falcón, 2008).

En este sentido, toma especial relevancia la calidad del

Dirección para correspondencia [Correspodence address]: Pilar Aparicio Chueca. Departamento de Empresa. Facultad de Economía y Empresa Universidad de Barcelona (España). E-mail: pilaraparicio@ub.edu servicio, que se ha definido desde diferentes perspectivas y ha sido uno de los grandes temas de debate en el área del marketing de los últimos ańos. El objetivo de toda organización es atraer y retener clientes, y proveerles de una alta calidad del servicio es crucial para conseguirlo. La calidad del servicio es un indicador clave de éxito de aquellas empresas que prosperan con una estrategia basada en la orientación al servicio (Rajaguru, 2016). De esta manera, la consecución de un servicio de calidad es uno de los medios con lo que cuentan las organizaciones para conseguir diferenciarse, en un mercado maduro y altamente competitivo como el de las instalaciones deportivas.

En cuanto a la definición del concepto, no existe una única aceptada universalmente (Sebastianelli y Tamini, 2002), a pesar de que resulta imprescindible para que pueda hablarse de gestión de la calidad, y en consecuencia sea posible diseñar un instrumento que permita su evaluación. La definición de Zeithaml (1988; p. 3) ha sido la más aceptada por los académicos, que expresa el término como "el juicio del consumidor sobre la excelencia o superioridad de un producto o de un servicio".

La importancia del sector del fitness a nivel europeo es notoria, dada su importancia en la contribución del deporte de forma positiva hacia el crecimiento europeo, la empleabilidad de los ciudadanos y la cohesión social, mientras que limita el gasto sanitario (European Comission, 2007). En este sentido, 
la práctica del deporte afecta a aspectos tan dispares como la salud, el estatus social o las relaciones afectivas, así como genera oportunidades en cuanto a servicios ofrecidos se refiere (Sánchez, 2011). La variedad de centros deportivos en el mercado, ofreciendo los mismos servicios, o similares, incrementa la importancia no solo de atraer al consumidor, sino de retenerlo.

De acuerdo con el informe Deloitte (2018) el sector europeo de la salud y el fitness atiende a 60 millones de consumidores y dispone de 59.055 centros especializados. Con un volumen de mercado de más de 26.600 millones de euros, se evidencia que Europa ha asumido el liderazgo, como el sector más grande a nivel mundial. Los cinco países líderes representan el 65\% del mercado total de la salud y el fitness en Europa (Alemania, Reino Unido, Francia, Italia y España).

El concepto de satisfacción del cliente ha sido conceptualizado como un componente crucial para el éxito de las organizaciones de fitness. Juntamente con éste término, Martínez y Martínez (2007) consideran que la calidad del servicio es un tema de interés en el área de la gestión, por su aplicabilidad en todas las áreas de gestión, y en especial del sector servicios. Mejorar la calidad del servicio que se ofrece a los clientes, sirve para mejorar su satisfacción (Lu, Tu y Jen, 2011). Asimismo cabe destacar que mientras que las evaluaciones de la calidad percibida son cognitivas en su mayoría, la satisfacción incluye evaluaciones post-consumo del servicio (Gallarza, Gil-Saura y Holbrook, 2011).

En el sector deportivo el análisis de la satisfacción del cliente ha sido estudiado tanto desde centros deportivos públicos o privados, como la asistencia de eventos deportivos (Calabuig, Molina y Nuñez, 2012; Martínez y Martínez, 2007; Murray y Howat, 2002; Van Leeuwen, Quick y Daniel, 2002). Howat, Murray y Crilley (1999) demostraron que la satisfacción de los clientes de los centros deportivos estaba positivamente relacionada con su intención de recomendar el servicio, de volver a utilizar el servicio y de incrementar la frecuencia de visitas, de manera similar a como suceden en otras empresas del sector servicios, aunque con particularidades.

La falta de consenso para establecer una definición de satisfacción única, ha generado discrepancias a la hora de medir el constructo. En algunos estudios en los que se analiza la satisfacción del consumidor, se ha utilizado un solo ítem para su valoración (Ferrand, Robinson y Valette-Florence, 2010; Ganesh, Arnold y Reynolds 2000; McCollough, Berry y Yadav, 2000; Murray y Howat, 2002). Posteriormente, y cuando se analizan los modelos que miden el efecto de la satisfacción sobre la fidelización empiezan a utilizarse escalas multidimensionales, utilizando combinaciones para la satisfacción general y la experiencia. Por ejemplo, Brady, Knight, Cronin Jr., Tomas, Hult, y Keillor (2005) utilizan una escala de tres ítems; Howat et al. (2008) una escala de dos ítems, en el que a la satisfacción general con el servicio se valora tam- bién la experiencia vivida por los consumidores; mientras que Li y Petrick (2010) utilizan una escala de cuatro ítems.

Del reciente estudio publicado por Aerobic\&Fitness (2018) sobre las Actividades Dirigidas (AADD) y su impacto sobre la satisfacción en los centros deportivos, surgió la necesidad de analizar a los usuarios que realizan actividades dirigidas en centros deportivos municipales para comparar el nivel de satisfacción entre los usuarios que realizan AADD de los que no las realizan. El estudio de Aerobic\&Fitness (2018) concluye que el $40 \%$ de los ingresos de los gimnasios en España son atribuibles a las actividades dirigidas. Además, el 63,3\% de los usuarios de centros deportivos asiste regularmente a las clases dirigidas, siendo las mujeres las que más participan, con un $77 \%$, mientras que los hombres lo hacen en un $45 \%$. Por otro lado, el 58,1\% de los usuarios abandonaría el centro si éste dejara de ofrecer el servicio de actividades dirigidas.

Baena et al. (2016) en su estudio acerca de las diferencias de satisfacción de los usuarios entre las actividades dirigidas con técnicos de las actividades dirigidas virtuales, concluye que las percepciones que tiene el cliente de las actividades dirigidas con técnico son significativamente mejores que las virtuales.

El objetivo de este estudio es el de conocer los factores que subyacen en el nivel de satisfacción de los usuarios de centros deportivos municipales que realizan actividades dirigidas y compararlo con aquellos usuarios que no hacen uso de este servicio.

\section{Método}

\section{Participantes}

Esta investigación recoge una encuesta realizada a usuarios de centros deportivos municipales de la ciudad de Barcelona, de un total de 42 con los que cuenta la ciudad. Los datos se recogieron durante los meses de abril a junio de 2013. El muestreo fue estratificado, de tipo intencional no probabilístico. El universo total lo componen 186.726 abonados, por lo que la muestra a estudiar con error del $\pm 3,1 \%$ para un nivel de confianza del $95 \%(Z=1,96)$ fue de 791 , de los cuales el $53 \%$ fueron hombres y el $47 \%$ mujeres. A su vez el $71 \%$ eran menores de 50 ańos, el 45,9\% tienen estudios superiores y el $71 \%$ llevaban como usuarios más de un año. De las 791 encuestas recogidas válidas, 536 sujetos $(67,7 \%$ del total) manifestaron realizar actividades dirigidas en el centro.

\section{Instrumento}

Se elaboró un cuestionario compuesto por una escala de 32 ítems que recogían atributos referentes a la calidad del servicio, la satisfacción, el valor percibido del servicio, las intenciones futuras y la realización y satisfacción con las actividades 
dirigidas en el centro. Los ítems fueron adoptados de estudios previos (Ganesh, Arnold y Reynolds, 2000; Murray y Howat, 2002; Parasuraman, Zeithaml y Berry, 1988; Triadó, Aparicio y Rimbau, 1999).

La escala utilizada para todas las preguntas es de tipo Likert, que oscilaba entre 1 (poco de acuerdo) a 10 (muy de acuerdo). El análisis de fiabilidad toma un valor correcto, con un alpha de Cronbach de .825 que permite garantizar el nivel de fiabilidad de la escala de medida.

\section{Procedimiento}

La muestra se recogió de forma aleatoria durante los meses de abril, mayo y junio de 2013, de 12 centros deportivos. El trabajo de campo se realizó mediante un cuestionario administrado con presencia del encuestador, que evitaba interpretaciones equivocadas a cada cuestión, respondiendo a las dudas que podían generar las preguntas. En algunos centros fueron ellos mismos quienes distribuyeron los cuestionarios entre sus usuarios, a través de los técnicos y personal auxiliar, mientras que en otros, dos personas del equipo se trasladaron para realizarlas. En el primer caso se hizo llegar una ficha técnica con las pautas necesarias para llevar a cabo la encuesta, donde se recomendaba, entre otros, pasar las encuestas en los diferentes horarios de funcionamiento del centro. En el segundo caso, las dos personas se colocaron en los lugares de mayor peso del centro (entrada y salida) y las encuestas se realizaban o bien mediante entrevista personal o bien lo hacían los mis- mos usuarios sentados en el área social del centro. El tiempo aproximado de respuesta fue de 10 minutos en promedio. Antes de proceder a la recogida de datos, se pidió permiso a el Institut Barcelona Esports, organismo autónomo del Ayuntamiento de Barcelona, con el encargo de gestionar el deporte en la ciudad, responsable de las instalaciones deportivas.

\section{Análisis estadístico}

Para el análisis de datos se utilizó el paquete informático SPSS24. Se llevó a cabo un análisis factorial exploratorio diferenciando aquellos usuarios que sí realizan actividades dirigidas $(\mathrm{N}=536)$, de aquellos que, siendo usuarios del centro, no las utilizan $(\mathrm{N}=217)$. Posteriormente, se realizó una regresión múltiple de los factores obtenidos, con el objetivo de estudiar el efecto de cada factor en la satisfacción del cliente de los usuarios que realizan actividades dirigidas y en los que no.

\section{Resultados}

Del total de la muestra, el $67,7 \%$ de los usuarios manifestaron realizar actividades dirigidas en el centro. El perfil sociodemográfico de estos clientes es de un $54,1 \%$ de mujeres, con estudios superiores, de los cuales el 48,9\% utilizan más habitualmente el horario de mañana. Estos usuarios tienen una satisfacción media con el centro de 7,8 puntos sobre diez (Tabla 1).

Tabla 1. Perfil sociodemográfico de los usuarios que realizan actividades dirigidas

\begin{tabular}{|c|c|c|c|c|c|}
\hline \multicolumn{2}{|c|}{ Distribución según género } & \multicolumn{2}{|c|}{ Distribución según el horario } & \multicolumn{2}{|l|}{ Nivel de estudios } \\
\hline Hombre & $45,9 \%$ & Mañana & $48,9 \%$ & Primarios & $7,6 \%$ \\
\hline \multirow[t]{4}{*}{ Mujer } & $54,1 \%$ & Mediodía & $11,6 \%$ & Secundarios & $10,5 \%$ \\
\hline & & Tarde & $35,3 \%$ & Bachillerato o $\mathrm{FP} 2^{\circ}$ grado & $35,8 \%$ \\
\hline & & Fines de semana & $4,2 \%$ & Diplomado o Ingeniero Técnico & $19,7 \%$ \\
\hline & & & & Licenciado, Ingeniero Superior & $26,4 \%$ \\
\hline
\end{tabular}

Con el objetivo de reducir las variables que contribuyen a la satisfacción, del análisis factorial se han obtenido tres facto- res, resultando una capacidad explicativa del 63,75\% de la varianza, como se muestra en la tabla 2. 
Tabla 2. Factores identificados en usuarios que realizan actividades dirigidas. Matriz de componentes rotados.

\begin{tabular}{|c|c|c|c|}
\hline Descripción & F1 & $\mathrm{F} 2$ & F3 \\
\hline El mantenimiento de las instalaciones & 0,721 & & \\
\hline La limpieza de las instalaciones & 0,616 & & \\
\hline Las instalaciones en conjunto & 0,695 & & \\
\hline Información sobre cambios en los servicios que utiliza actualmente & 0,805 & & \\
\hline Información de propuestas de nuevos servicios por parte del centro & 0,794 & & \\
\hline El trato humano que recibe de los profesionales técnicos & & 0,850 & \\
\hline El nivel formativo de los profesionales técnicos & & 0,841 & \\
\hline El trato que recibe del personal auxiliar & & 0,673 & \\
\hline Práctica del deporte para hacer amigos & & & 0,847 \\
\hline Importancia de hacer amigos en el centro & & & 0,848 \\
\hline $\begin{array}{l}\text { Varianzas explicadas } \\
K M O=0,788 \\
\text { Bartlett's test p-value, } 000 \\
\text { Varianza total explicada }=63,75 \%\end{array}$ & $25,77 \%$ & $22,53 \%$ & $15,45 \%$ \\
\hline
\end{tabular}

F1: Instalaciones y Comunicación; F2: Recursos Humanos; F3: Entorno y clima social.

El primer factor recoge las variables referentes a la calidad de las instalaciones y a la comunicación del centro. Explica el 25,77\% e incorpora variables de mantenimiento, limpieza de las instalaciones, e información de los servicios que ofrece el centro. El segundo factor agrupa las variables referentes a los recursos humanos. Explica alrededor del 22\% del modelo e incluye la profesionalidad percibida del cliente hacia los empleados técnicos, y la del trato recibido tanto del personal técnico como auxiliar. El tercer factor, que explica el $15,45 \%$ de la varianza recoge las variables relacionadas con el entorno y el clima social que crean los clientes de una organización. Expresa el interés y la importancia de hacer amigos, en este caso, practicando deporte.
Posteriormente, se ha realizado una regresión múltiple de los factores obtenidos con anterioridad, con el objetivo de estudiar el efecto de cada factor en la satisfacción del cliente de una instalación deportiva. Para los usuarios que realizan actividades dirigidas, el modelo de regresión tiene una $\mathrm{R}^{2}=$ 0,543 con un error típico de estimación de 1,03. El modelo se corrobora estadísticamente por el valor obtenido por el estadístico $\mathrm{F}$ y es apropiado para la explicación de la satisfacción global del cliente (Tabla 3). Los valores del estadístico t muestran que todos los factores son significativos.

Tabla 3. Modelo de regresión múltiple. Usuarios que sí realizan actividades dirigidas. Coeficientes de regresión

\begin{tabular}{|c|c|c|c|c|c|}
\hline & \multicolumn{2}{|c|}{ Coeficientes no Estandarizados } & \multirow{2}{*}{$\frac{\text { Coeficientes tipificados }}{\text { Beta }}$} & \multirow[b]{2}{*}{$\mathbf{t}$} & \multirow{2}{*}{ Sig. } \\
\hline & B & Error tip. & & & \\
\hline Constante & 7,846 & 044 & & 176,628 & ,000 \\
\hline (F1) Calidad Instalaciones y comunicación & 918 & ,044 & ,605 & 20,705 & 000 \\
\hline (F2) Recursos humanos & ,622 & 044 & ,409 & 13,982 &, 000 \\
\hline (F3) Entorno social &, 151 &, 044 &, 100 & 3,412 & ,001 \\
\hline
\end{tabular}

En el modelo la variable calidad de las instalaciones y comunicación fue el mejor predictor de la satisfacción, con un valor beta de 0,605 , mientras que el entorno social presentó el valor beta más bajo, con un 0,100 .

El modelo resultante es significativo y se resume en la siguiente ecuación:

$$
\text { satisfacción }=7,846+0,605 F 1+0,409 F 2+0,100 F 3+e_{i}
$$

donde:

F1: Calidad de las instalaciones y comunicación

F2: Recursos humanos

F3: Entorno y clima social

Para los usuarios que no realizan actividades dirigidas, el número de factores es de tres, igual que en el anterior, pero la composición de cada uno de ellos es diferente. En este caso el primer factor está formado por variables de recursos 
humanos y comunicación, con una varianza del $24,27 \%$, el segundo factor por las instalaciones, explicado por el 23,06\% de la varianza y el tercer factor por el entorno social y la rela- ción calidad-precio, con un 16,19\%. Esta última variable en el caso de los usuarios de actividades dirigidas no es significativa (tabla 4).

Tabla 4. Factores identificados en usuarios que no realizan actividades dirigidas. Matriz de componentes rotados

\begin{tabular}{|c|c|c|c|}
\hline Descripción & F1 & F2 & F3 \\
\hline El nivel formativo de los profesionales técnicos & 0,772 & & \\
\hline El trato humano que recibe de los profesionales técnicos & 0,748 & & \\
\hline El trato que recibe del personal auxiliar & 0,540 & & \\
\hline Información sobre cambios en los servicios que utiliza actualmente & 0,677 & & \\
\hline Información de propuestas de nuevos servicios por parte del centro & 0,692 & & \\
\hline Las instalaciones en conjunto & & 0,807 & \\
\hline La limpieza de las instalaciones & & 0,904 & \\
\hline El mantenimiento de las instalaciones & & 0,902 & \\
\hline Práctica del deporte para hacer amigos & & & 0,809 \\
\hline Importancia de hacer amigos en el centro & & & 0,734 \\
\hline Relación calidad-precio & & & 0,657 \\
\hline $\begin{array}{l}\text { Varianzas explicadas } \\
K M O=0,715 \\
\text { Bartlett's test p-value,000 } \\
\text { Varianza total explicada }=63,52 \%\end{array}$ & $24,27 \%$ & $23,06 \%$ & $16,19 \%$ \\
\hline
\end{tabular}

Para este segundo grupo de usuarios, el modelo de regre- error típico de estimación de 1,08 (tabla 5). sión resultante es significativo y obtiene una $\mathrm{R}^{2}=0,47$, con un

Tabla 5. Modelo de regresión múltiple. Usuarios que no realizan actividades dirigidas. Coeficientes de regresión

\begin{tabular}{|c|c|c|c|c|c|}
\hline & \multicolumn{2}{|c|}{ Coeficientes no Estandarizados } & \multirow{2}{*}{$\frac{\text { Coeficientes tipificados }}{\text { Beta }}$} & \multirow{2}{*}{$\mathbf{t}$} & \multirow{2}{*}{ Sig. } \\
\hline & B & Error tip. & & & \\
\hline Constante & 7,683 & , 080 & & 95,670 & 000 \\
\hline (F1) Recursos humanos y comunicación &, 571 & ,081 & ,383 & 7,092 &, 000 \\
\hline (F2) Instalaciones & ,829 &, 081 &, 555 & 10,292 &, 000 \\
\hline (F3) Entorno social y calidad-precio & ,234 & 081 & 157 & 2,910 &, 004 \\
\hline
\end{tabular}

El modelo resultante es significativo y se resume en la siguiente ecuación:
satisfacción $=7,683+0,383 F 1+0,555 F 2+0,157 F 3+e$
donde:
F1: Recursos humanos y comunicación
F2: Instalaciones
F3: Entorno social y calidad-precio

\section{Discusión y conclusiones}

El objetivo del presente estudio fue el de conocer los factores que subyacen en el nivel de satisfacción de los usuarios de centros deportivos municipales que realizan actividades dirigidas y compararlo con aquellos usuarios que no hacen uso de este servicio.
Los resultados sugieren que los usuarios de actividades dirigidas en centros deportivos tienen una satisfacción media con el centro bastante notable, con una puntuación media de 7,8 puntos sobre un máximo de 10 , cuyo resultado demuestra una valoración general positiva por parte de los usuarios, al igual que los resultados hallados en los estudios previos de Nuviala, Grao-Cruces, Pérez-Turpin y Nuviala (2012) y Rial et al. (2010). Acorde con Elasri, Triadó y Aparicio (2015) y Nuviala et al. (2012) las variables relacionadas con los recursos humanos fueron las mejor valoradas por los clientes, mientras que la variable de hacer amigos en el centro es con la que se sienten menos satisfechos, a pesar de ser uno de los factores que el cliente emplea para valorar el centro deportivo.

Según las características sociodemográficas, y acorde con el trabajo de Baena et al., 2016 y el estudio elaborado por 
Aerobic\&Fitness (2018), las mujeres y los usuarios con estudios medios son los que mayor uso hacen de éstas. Uno de los segmentos encontrados por el trabajo de Elasri, Triadó y Aparicio (2016) en su análisis de la segmentación de usuarios en centros deportivos municipales, fue el "old-social", compuesto de manera principal por mujeres mayores de 60 ańos, que daban especial importancia a las relaciones sociales en el centro. Este hecho pueden explicar los hallazgos sociodemográficos encontrados en el presente estudio, ya que las actividades dirigidas permiten sociabilizarse con un mayor número de personas, siendo una de las razones por las cuales las mujeres acuden a un centro deportivo.

De todas las variables que se pueden emplear para valorar una instalación deportiva, para los usuarios de actividades dirigidas se reducen a tres factores: instalaciones y comunicación, recursos humanos y entorno social. El mismo número de factores es el que aparece para los usuarios que no utilizan el servicio de las actividades dirigidas, aunque la composición de los factores es diferente. En este caso, los factores son: recursos humanos y comunicación, instalaciones y entorno social y calidad-precio.

La comunicación toma un papel relevante en ambos tipos de clientes, como consecuencia del esfuerzo por parte de los gerentes para llegar a todos los clientes de la manera más rápida y eficiente posible (Elasri, Triadó y Aparicio, 2015). Las redes sociales se han convertido en un método masivo de comunicación, con el objetivo de construir relaciones más sólidas con el cliente (Yoo, Lee y Bai, 2010). Según Agencia Visual (2017) el 97\% de los usuarios de internet buscan en la red la información sobre marcas y productos, por lo que las herramientas de social media son indispensables para cualquier empresa. Estas herramientas aplicadas como estrategia de comunicación en los centros deportivos pueden establecer una relación más cercana y cotidiana de los usuarios hacia el centro, consiguiendo mayores recomendaciones de éstos a terceros. Por otro lado, este tipo de estrategia requiere una menor inversión frente al marketing tradicional, permitiendo alargar un conjunto de acciones en el tiempo, y testar las estrategias con las que se conseguirá adaptar a la demanda de los seguidores. Algunas de las redes sociales que mayores posibilidades ofrecen son: Facebook, Twitter, Instagram, Youtube, LinkedIn, Pinterest, entre otros.

Finalmente, se observa en que el mejor predictor de la satisfacción, en ambos casos, es el factor que engloba las variables referentes a las instalaciones, aunque en el caso de los usuarios que no utilizan el servicio, no se tienen en cuenta las variables de comunicación. Esto puede venir explicado, debido a que los clientes que utilizan las actividades dirigidas son las que necesitan están más conectadas con el centro, debido al seguimiento que puede hacerse en el desarrollo de la actividad dirigida, o bien por posibles cambios de horario. El entorno social, en ambos tipos de usuarios, presentaron el valor más bajo como predictor de la satisfacción.

Las limitaciones de este estudio, que motivan futuras investigaciones, hacen referencia al cuestionario, ya que se procedió a realizarlo de forma genérica a todos los usuarios de los centros deportivos mencionados. Sería interesante trabajar exclusivamente con aquellos usuarios que realizan actividades dirigidas en el centro y poder analizar otras variables además de la satisfacción, como las intenciones futuras.

\section{Referencias}

1. Aerobic\&Fitness (2018). Actividades dirigidas. Su inversión e impacto en el centro deportivo. Análisis de la oferta y la demanda. Recuperado de: https://www.aerobicyfitness.com/content/24-estudio-actividadesdirigidas

2. Agencia Visual (2017). Las redes sociales como parte de la estrategia de comunicación de la empresa. Recuperado de: http://agenciavisual.es/ redes-sociales-comunicacion/

3. Baena-Arroyo, J., García-Fernández, J., Bernal-García, A., Lara-Bocanegra, A., y Gálvez-Ruíz, P. (2016). El valor percibido y la satisfacción del cliente en actividades dirigidas virtuales y con técnico en centros de fitness. Revista de psicología del deporte, 25(2).

4. Brady, M., Knight, G., Cronin Jr., J., Tomas, G., Hult, M., y Keillor, B. (2005). Removing the contextual lens: A multinational, multi-setting comparison of service evaluation models. Journal of Retailing, 81(3), 215-230.

5. Calabuig, F., Molina, N. M., y Núñez-Pomar, J. (2012). Una aplicación inicial del modelo tridimensional de calidad de servicio en centros deportivos privados. E-Balonmano.com: Revista de Ciencias Del Deporte, 8(1), 67-81.

6. Celestino, A., y Biencinto, C. (2012). La satisfacción del cliente externo en organizaciones de fitness. Estudio empírico en centros de la Comunidad de Madrid. Motricidad. European Journal of Human Movement, 29.

7. Cronin, J. J., Brady, M. K., y Hult, G. T. M. (2000). Assessing the effects of quality, value, and customer satisfaction on consumer behavioral intentions in service environments. Journal of Retailing, 76(2), 193-218.

8. Deloitte. (2018). European Health y Fitness Market 2016. Recuperado de: https://www2.deloitte.com/content/dam/Deloitte/de/Documents/ consumer-business/European $\% 20$ Health\%20and $\% 20$ Fitness $\% 20$ Report_2018_extract.pdf

9. Elasri, A., Triadó, X. M., y Aparicio, P.(2015). La satisfacción de los clientes de los centros deportivos municipales de Barcelona. Apunts. Educació Física i Esports, (119), 109-117.

10. Elasri-Ejjaberi, A., Triadó-Ivern, X. M., y Aparicio-Chueca, P. (2016) Los usuarios de los centros deportivos públicos: una aproximación de segmentación sobre hábitos deportivos y satisfacción. Revista de Psicología del Deporte, 25(1).

11. European Comission (2007). White paper on strategy for Europe on nutrition, overweight and obesity related health issues. Brussels, Belgium.

12. Ferrand, A., Robinson, L., y Valette-Florence, P. (2010). The Intention-to-Repurchase Paradox: A Case of the Health and Fitness Industry. Journal of Sport Management, 24, 83-105.

13. Ganesh, J., Arnold, M. J., y Reynolds, K. E. (2000). Understanding the Customer Base of Service Providers: An Examination of the Differences Between Switchers and Stayers. Journal of Marketing, 64(3), 65-87.

14. Howat, G., Crilley, G., y Mcgrath, R. (2008). A focused service quality, 
benefits, overall satisfaction and loyalty model for public aquatic centres. Managing Leisure, 13(3-4), 139-161.

15. Howat, G., Murray, D., y Crilley, G. (1999). The Relationships between Service Problems and Perceptions of Service Quality, Satisfaction, and Behavioral Intentions of Australian Public Sports and Leisure Center Customers. Journal of Park and Recreation Administration, 17(2), 42-64.

16. Li, X., y Petrick, J. F. (2010). Towards an Integrative Model of Loyalty Formation: The Role of Quality and Value. Leisure Sciences, 32(3), 201-221.

17. Martínez, L., y Martínez, J. A. (2007). Cognitive-affective model of consumer satisfaction. An exploratory study within the framework of a sporting event. Journal of Business Research, 60(2), 108-114.

18. McCollough, M. A., Berry, L. L., y Yadav, M. S. (2000). An Empirical Investigation of Customer Satisfaction after Service Failure and Recovery. Journal of Service Research, 3(2), 121-137.

19. Murray, D., y Howat, G. (2002). The Relationships among Service Quality, Value, Satisfaction, and Future Intentions of Customers at an Australian Sports and Leisure Centre. Sport Management Review, 5(1), $25-43$.

20. Nuviala, A., Tamayo, J. A., Iranzo, J., y Falcón, D. (2008). Creación, diseńo, validación y puesta en práctica de un instrumento de medición de la satisfacción de usuarios de organizaciones que prestan servicios deportivos. Retos. Nuevas tendencias en Educación Física, Deporte y Recreación, 14(2), 10-16.
21. Nuviala, A., Grao-Cruces, A., Pérez-Turpin, J. A., y Nuviala, R. (2012). Perceived service quality, perceived value and satisfaction in groups of users of sports organizations in Spain. Kinesiology, 44(1), 94-103.

22. Rajaguru, R. (2016). Role of value for money and service quality on behavioural intention: A study of full service and low-cost airlines. Journal of Air Transport Management, 53, 114-122.

23. Rial-Boubeta, J., Varela-Mallou, J., Rial-Boubeta, A., y Real-Deus, E. (2010). Modelización y medida de la Calidad Percibida en centros deportivos: la escala QSport-10. RICYDE. Revista Internacional de Ciencias del Deporte, 6(18), 57-73.

24. Sánchez, J. (2011). Business y Fitness: El negocio de los centros deportivos: Editorial UOC.

25. Sebastianelli, R., y Tamimi, N. (2002). How product quality dimensions relate to defining quality. International Journal of Quality y Reliability Management, 19(4), 442-453.

26. Van Leeuwen, L., Quick, S., y Daniel, K. (2002). The Sport Spectator Satisfaction Model: A Conceptual Framework for Understanding the Satisfaction of Spectators. Sport Management Review, 5(2), 99-128.

27. Yoo, M., Lee, S., y Bai, B. (2011). Hospitality marketing research from 2000 to 2009: topics, methods, and trends. International Journal of Contemporary Hospitality Management, 23(4), 517-532.

28. Zeithaml, V. A. (1988). Consumer Perceptions of Price, Quality, and Value: A Means-End Model and Synthesis of Evidence. Journal of Marketing, 52(3), 2-22. 